\title{
The Convective Instabilities in a Liquid-Vapor System with a Non-equilibrium Evaporation Interface
}

\author{
Rong Liu • Qiusheng Liu
}

Received: 12 February 2009 / Accepted: 25 May 2009 / Published online: 10 June 2009

(C) Springer Science + Business Media B.V. 2009

\begin{abstract}
Rayleigh-Marangoni-Bénard instability in a system consisting of a horizontal liquid layer and its own vapor has been investigated. The two layers are separated by a deformable evaporation interface. A linear stability analysis is carried out to study the convective instability during evaporation. In previous works, the interface is assumed to be under equilibrium state. In contrast with previous works, we give up the equilibrium assumption and use Hertz-Knudsen's relation to describe the phase change under non-equilibrium state. The influence of Marangoni effect, gravitational effect, degree of non-equilibrium and the dynamics of the vapor on the instability are discussed.
\end{abstract}

Keywords Rayleigh-Marangoni-Bénard • Instability • Evaporation

\section{Introduction}

Convection occurring in an horizontal liquid layer has received extensive attention (Rayleigh 1916; Block 1956; Pearson 1958; Nield 1964) since Bénard (1900) observed hexagonal roll cells upon onset of convection in molten spermaceti with a free surface. A more complicated problem is the Rayleigh-Marangoni con-

R. Liu · Q. Liu ( $\bowtie)$

Key Laboratory of Microgravity (National

Microgravity Laboratory), Institute of Mechanics,

Chinese Academy of Sciences,

Beijing 100190, China

e-mail: liu@imech.ac.cn

R. Liu

e-mail: liurong@imech.ac.cn vection in a liquid layer with an evaporation interface. Evaporative convection is of great interest in engineering because of its importance in modern technologies such as thin-film evaporators, boiling equipments and heat pipes. During evaporation, an essential mechanism is that evaporation leads to intensive cooling of the liquid-vapor interface. When the temperature drop induced by evaporation across the liquid layer exceeds a critical value, convective instability occurs. This instability has been studied by many previous investigators. Miller (1973) examined the instabilities of an isothermal evaporating interface associated with a moving boundary. Prosperetti and Plesset (1984) studied the stability of an evaporating liquid surface. In their analysis, the viscosity of the vapor and the liquid is neglected and the depth of the vapor and the liquid is infinite. Palmer (1976) investigated the hydrodynamic stability of rapidly evaporating liquids at reduced pressure in an infinite depth liquid-vapor system. Burelbach et al. (1988) investigated the nonlinear stability of evaporating and condensing liquid films. Vapor recoil, thermocapillary and rupture instabilities are discussed in their works.

In most of previous works, studies on convective instability were carried out for one-side models, and the vapor phase adjacent to the liquid layer was considered to be passive or infinitely deep. In this case, the dynamics of vapor was assumed to have little influence on the instability of the liquid layer, consequently the dynamics of the vapor was neglected. Ozen and Narayanan (2004) question the assumption that the vapor is passive. They suggested that the active vapor layer plays a major role in determining the stability of system. They proposed a two-side model and focused on the physics of evaporative instability taking into account the effect 
of a finite-depth active vapor. In their model, interfacial chemical potential equilibrium assumption is used, i.e., the temperature of the liquid is at its saturation value with respect to the vapor pressure. This assumption is questionable. Indeed, a more general relation like the Hertz-Knudsen relation (Colinet et al. 2001) should be used in place of the chemical potential equilibrium assumption.

In previous literatures, it is conventional to require thermal equilibrium between liquid and vapor (continuity of temperature) at the interface. However, a temperature discontinuity at the liquid-vapor interface has been reported by several investigators. Moreover, Fang and Ward (1999) have confirmed the existence of interfacial temperature discontinuity by experiments. Margerit et al. (2003) used new interfacial constitutive relations to describe the interfacial heat and mass transfer. Several interfacial coefficients was used in their analysis. These relations generalize the interfacial nonequilibrium and temperature discontinuity. However, the interfacial coefficients was only determined for water at $0^{\circ} \mathrm{C}$. So, such a model is difficult to be used for other systems.

In this paper, we focus on the non-equilibrium effect of evaporation. For this reason, we neglect the temperature discontinuity as most of early works. The goal of this paper is to construct a relatively more rigorous analysis without making assumptions in which the vapor is passive and the interface is under interfacial chemical equilibrium.

The structure of this paper is the following. In "Mathematical Model" the governing equations are established, and boundary conditions taking into account the interfacial non-equilibrium effect are discussed in detail. In "Numerical Method", a linear stability analysis is presented. In "Results and Discussion", the influence of physical parameters on the instability of the system is discussed. Finally, conclusions are drawn in "Conclusion".

\section{Mathematical Model}

We propose a two layer model with a deformable interface, as shown in Fig. 1. The system consists of a liquid of depth $d_{l}$ underling its own vapor of depth $d_{v}$. The system is infinite in the horizontal directions. The top wall and the bottom wall are considered as rigid perfectly conducting boundaries. The top wall is a porous medium, through which the vapor can be evacuated. The phase change rate at the interface can be controlled by a pump that can adjust the vapor pressure. The depth of the liquid is assumed constant by suitably

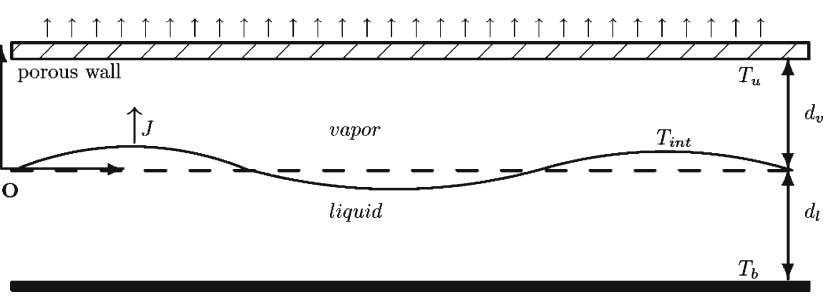

Fig. 1 Physical model

adjusting the liquid feed back. In unperturbed state, the liquid is evaporating at a constant rate. However, the local evaporating velocity is not constant as it can change upon perturbation. Hertz-Knudsen's equation (Kennard 1938; Colinet et al. 2001) predicts a mass flux at the interface.

\section{Governing Equations and Boundary Conditions}

The fluid is assume to satisfy the Boussinesq approximation, i.e., all fluid properties are assumed to be independent of temperature, except density which decreases linearly with the temperature. The governing equations for each fluid layer are the continuity equation, the Navier-Stokes equation and the energy equation:

$\nabla \cdot \mathbf{u}_{i}=0$

$$
\begin{aligned}
\frac{\partial \mathbf{u}_{i}}{\partial t} & +\mathbf{u}_{i} \cdot \nabla \mathbf{u}_{i} \\
& =-\frac{1}{\rho_{i}} \nabla p_{i}+\left[1-\beta_{i}\left(T_{i}-T_{0}\right)\right] \mathbf{g}+v_{i} \nabla^{2} \mathbf{u}_{i}
\end{aligned}
$$

$\frac{\partial T_{i}}{\partial t}+\mathbf{u}_{i} \cdot \nabla T_{i}=\kappa_{i} \nabla^{2} T_{i}$

In the equations above, $\mathbf{u}, p$ and $T$ are velocity, pressure and temperature. $\rho, v, \kappa$ are the density, kinematic viscosity, and the thermal diffusivity. The subscript $i=$ $v, l$ denote the vapor and the liquid, respectively. Note that $\rho$ is the density at a certain reference temperature. Further more, the variation of the density is only taken into account in the body force term of the NavierStokes equation.

The bottom and top walls are perfectly conducting boundaries, so that

$T_{v}\left(d_{v}\right)=T_{u}, T_{l}\left(-d_{l}\right)=T_{b}$

The no-slip condition along the bottom plate:

$u_{l}\left(-d_{l}\right)=0, w_{l}\left(-d_{l}\right)=0$

here $u$ and $w$ are the horizontal and vertical components of $\mathbf{u}$. The permeable condition of the vertical 
velocity and no-slip condition of the horizontal velocity along the top plate:

$u_{v}\left(d_{v}\right)=0, w_{v}\left(d_{v}\right)=w_{v 0}$

At the deformable interface, the position of the interface can be described by its variable height $z=$ $\eta(x, t)$. If $\mathbf{u}_{\text {int }}$ denotes the interfacial velocity, a kinematic relation between $\mathbf{u}_{i n t}$ and $\eta(x, t)$ is obtained by $w_{\text {int }}=\mathrm{d} z / \mathrm{d} t$ and $u_{\text {int }}=\mathrm{d} x / \mathrm{d} t$. Thus

$w_{\text {int }}=\frac{\partial \eta}{\partial t}+u_{\text {int }} \frac{\partial \eta}{\partial x}$

The mass balance equation at the interface $z=\eta(x, t)$ is

$J=\rho_{v}\left(\mathbf{u}_{v}-\mathbf{u}_{i n t}\right) \cdot \mathbf{n}=\rho_{l}\left(\mathbf{u}_{l}-\mathbf{u}_{i n t}\right) \cdot \mathbf{n}$

Here $J$ is the evaporation flux.

The normal momentum and the tangential momentum balance equations at the interface are:

$J[\mathbf{u}]_{v}^{l} \cdot \mathbf{n}+[p-\mathbf{P} \cdot \mathbf{n} \cdot \mathbf{n}]_{v}^{l}=-2 \sigma H$

$[\mathbf{P} \cdot \mathbf{n} \cdot \mathbf{t}]_{v}^{l}=\frac{1}{N} \frac{\partial \sigma}{\partial T}\left(\frac{\partial T}{\partial x}+\frac{\partial \eta}{\partial x} \frac{\partial T}{\partial z}\right)$

here $N=\left[1+(\partial \eta / \partial x)^{2}\right]^{1 / 2} . \sigma$ is the surface tension. In general, the surface tension is assumed to depend on temperature only. $\sigma=\sigma_{0}-\sigma_{T}\left(T-T_{0}\right) .-\sigma_{T}$ is the surface tension variation with temperature.

The energy balance equation at the interface is:

$$
\begin{aligned}
& J\left[\left|\mathbf{u}-\mathbf{u}_{\text {int }}\right|^{2}\right]_{v}^{l}+q_{l}-q_{v}-\left[\mathbf{P} \cdot\left(\mathbf{u}-\mathbf{u}_{\text {int }}\right) \cdot \mathbf{n}\right]_{v}^{l} \\
& \quad=J L
\end{aligned}
$$

Here $L$ is the latent heat, $q$ is the heat conduction, $\mathbf{P}$ is the viscous strain tensor with the components $P_{i, j}=\mu\left(\frac{\partial u_{i}}{\partial x_{j}}+\frac{\partial u_{j}}{\partial x_{i}}\right) . q$ is the heat flux defined as $\chi \mathbf{n} \cdot \nabla T$, in which $\chi$ is the thermal conductivity. $[f]_{v}^{l}$ denotes $f_{l}-f_{v}$.

The temperatures are continuous at the interface:

$T_{v}=T_{l}$

The Hertz-Knudsen relation describes the evaporation flux and the difference between the saturate pressure and the vapor pressure at the interface:

$J=\beta \sqrt{\frac{M}{2 \pi R T}}\left[p_{s}(T)-p_{v}\right]$

Here $\beta$ is the evaporation accommodation coefficient, $M$ is the molecular weight of vapor, $p_{s}(T)$ is the saturation pressure at interfacial temperature $T, p_{v}$ is the vapor pressure just beyond the interface, $R$ is the universal gas constant.
The relation between the saturate pressure and interfacial temperature is described by the ClausiusClapeyron relation:

$p_{s}(T)=p_{0} \exp \left[\frac{L}{R}\left(\frac{1}{T}-\frac{1}{T_{0}}\right)\right]$

The tangential velocities of the liquid and vapor layer are equal:

$\mathbf{u}_{v} \cdot \mathbf{t}=\mathbf{u}_{l} \cdot \mathbf{t}$

In these equations, $\mathbf{n}$ is the unit normal vector, $\mathbf{t}$ is the unit tangential vector.

$\mathbf{n}=-\frac{-\frac{\partial \eta}{\partial x} \mathbf{e}_{x}+\mathbf{e}_{z}}{\left(1+\left(\frac{\partial \eta}{\partial x}\right)^{2}\right)^{1 / 2}}$

$\mathbf{t}=\frac{\mathbf{e}_{x}+\frac{\partial \eta}{\partial x} \mathbf{e}_{z}}{\left(1+\left(\frac{\partial \eta}{\partial x}\right)^{2}\right)^{1 / 2}}$

$2 H$ is the surface mean curvature.

$2 H=\frac{\frac{\partial^{2} \eta}{\partial x^{2}}}{\left[1+\left(\frac{\partial \eta}{\partial x}\right)^{2}\right]^{3 / 2}}$

Unperturbed State Solution of the System

In the unperturbed state there is no flow in the liquid layer and the evaporation rate is a constant, i.e. $u_{l 0}=$ $w_{l 0}=0$ and $u_{v 0}=0, w_{v 0}=J_{0} / \rho_{v}$. Here, the subscript 0 denotes the unperturbed state. The unperturbed temperature fields satisfy these equations:

$\kappa_{v} \frac{d^{2} T_{v 0}}{d z^{2}}=w_{v 0} \frac{d T_{v 0}}{d z}$

$\frac{d^{2} T_{l 0}}{d z^{2}}=0$

From these equations, we obtain:

$T_{v 0}=C_{1} \exp \left[\frac{w_{v 0}}{\kappa_{v}} z\right]+C_{2}, T_{l 0}=C_{3} z+C_{4}$

at the bottom wall $z=-d_{l}$ :

$T_{l}=T_{b}$

at the interface $z=0$ :

$T_{v 0}=T_{l 0}=T_{i n t}$

$-J_{0} L+\chi_{v} \frac{d T_{v 0}}{d z}=\chi_{l} \frac{d T_{l 0}}{d z}$ 
Given the temperature at the interface and the bottom wall, we can decide the coefficients $C_{1}, C_{2}, C_{3}, C_{4}$.

$$
\begin{aligned}
C_{1} & =\frac{\kappa_{v}}{\chi_{v} w_{v 0}}\left(J_{0} L+\chi_{l} \frac{T_{i n t}-T_{b}}{d_{l}}\right) \\
C_{2} & =T_{i n t}-\frac{\kappa_{v}}{\chi_{v} w_{v 0}}\left(J_{0} L+\chi_{l} \frac{T_{i n t}-T_{b}}{d_{l}}\right) \\
C_{3} & =\frac{T_{i n t}-T_{b}}{d_{l}}, C_{4}=T_{i n t}
\end{aligned}
$$

\section{Perturbation Equations}

To nondimensionalize the controlling equations, we chose the following scales $v_{l} / d_{l}, d_{l}^{2} / v_{l}, d_{l}$ and $\Delta T$ for velocity, time, length and temperature, respectively. Here $\Delta T=T_{b}-T_{\text {int }}$. The perturbations of velocity, pressure and temperature are decomposed into normal modes proportional to $\exp [\lambda t+i k x]$. Here $\lambda$ is the time growth rate, $k$ is the wave number. Using $U_{i}, W_{i}, \Theta_{i}$ and $P_{i}$ are the amplitudes of horizontal velocity, vertical velocity, temperature and pressure, and introducing spatial normal perturbations into the linearized full governing equations and boundary conditions, we obtain the normal mode equations in dimensionless forms:

$$
i k U_{v}+D W_{v}=0
$$

$$
\lambda \rho^{*} U_{v}+w_{v 0} D U_{v}=-i k P_{v}+v^{*} \rho^{*} \nabla^{2} U_{v}
$$

$$
\begin{aligned}
& \lambda \operatorname{Pr}^{*} W_{v}+\operatorname{Pr}_{v 0} D W_{v}= \\
& \quad-\operatorname{Pr} D P_{v}+\operatorname{Pr} v^{*} \rho^{*} \nabla^{2} W_{v}+\rho^{*} \beta^{*} R a \Theta_{v}
\end{aligned}
$$

$\lambda \operatorname{Pr} \Theta_{v}+\operatorname{Pr} \frac{\partial T_{v 0}}{\partial z} W_{v}+\operatorname{Pr} w_{v 0} D \Theta_{v}=\kappa^{*} \nabla^{2} \Theta_{v}$

$$
i k U_{l}+D W_{l}=0
$$

$$
\lambda U_{l}=-i k P_{l}+\nabla^{2} U_{l}
$$

$\lambda \operatorname{Pr} W_{l}=-\operatorname{Pr} D P_{l}+\operatorname{Pr} \nabla^{2} W_{l}+R a \Theta_{l}$

$$
\lambda \operatorname{Pr} \Theta_{l}+\operatorname{Pr} \frac{\partial T_{l 0}}{\partial z} W_{l}=\nabla^{2} \Theta_{l}
$$

Boundary conditions at the top wall $(z=h)$ :

$U_{v}=W_{v}=\Theta_{v}=0$

and the bottom wall $(z=-1)$ :

$U_{l}=W_{l}=\Theta_{l}=0$

Boundary conditions at the interface $(z=0)$ :

$\rho^{*}\left(W_{v}-\lambda \eta\right)=W_{l}-\lambda \eta$

$2 J_{0}\left(W_{l}-W_{v}\right)+P_{l}-P_{v}-2 D W_{l}+2 \mu^{*} D W_{v}$

$$
=\left[k^{2} \frac{1}{P r C a}+\left(1-\rho^{*}\right) \frac{G a}{P r}\right] \eta
$$

$\operatorname{Pr}\left(D U_{l}+i k W_{l}\right)-\operatorname{Pr} \mu^{*}\left(D U_{l}+i k W_{l}\right)$

$$
=-i k M a\left(\Theta_{l}+\frac{d T_{l 0}}{d z} \eta\right)
$$

$E\left(W_{l}-\lambda \eta\right)-\chi^{*} \frac{d \Theta_{v}}{d z}+\frac{d \Theta_{l}}{d z}=0$

$U_{l}=U_{v}$

$\Theta_{l}+\frac{\partial T_{l 0}}{\partial z} \eta=\Theta_{v}+\frac{\partial T_{v 0}}{\partial z} \eta$

$W_{v}-W_{l}=E_{2}\left[E_{1}^{-1}\left(\Theta_{l}+\frac{\partial T_{l 0}}{\partial z} \eta\right)-P_{v}\right]$

The depth ratio is $h=d_{v} / d_{l}$. The dimensionless ratio of the physical properties are $\kappa^{*}=\kappa_{v} / \kappa_{l}$ (thermal diffusivity), $\beta^{*}=\beta_{v} / \beta_{l}$ (volumetric expansion coefficient), $\chi^{*}=\chi_{v} / \chi_{l}$ (thermal conductivity), $\mu^{*}=\mu_{v} / \mu_{l}$ (dynamic viscosity), $\rho^{*}=\rho_{v} / \rho_{l}$ (density) and $v^{*}=v_{v} / v_{l}$ (kinematical viscosity), respectively. The subscripts $v$ and $l$ refer to the vapor and liquid layers respectively. $D$ is the dimensionless differential operator $d / d z, \nabla^{2}$ the operator $D^{2}-k^{2}$, and $d T_{i 0} / d z$ the temperature gradient of fluid- $i$ in the unperturbed state. $w_{v 0}$ is the dimensionless evaporation velocity in the unperturbed state. Ma is the Marangoni number defined as $\sigma_{T} \Delta T d_{l} /\left(\mu_{l} \kappa_{l}\right)$, $C a$ is the capillary number defined as $C a=\mu_{l} \kappa_{l} / \sigma_{0} d_{l}$, $G a$ is defined as $G a=g d_{l}^{3} / \nu_{l} \kappa_{l}, E$ is defined as $E=$ $\frac{\rho_{l} \nu_{l} L}{\chi_{l} \Delta T}, E_{1}$ is defined as $E_{1}=\frac{\rho_{l} \nu_{l}^{2} T_{0}}{\rho_{v} h_{l}^{2} L \Delta T}, E_{2}$ is defined as $E_{2}=\beta \sqrt{\frac{M}{2 \pi R T}} \frac{\rho_{l}-\rho_{v}}{\rho_{v}} \frac{\nu_{l}}{h_{l}}, \operatorname{Pr}$ is the Prandtl number defined as $\nu_{l} / \kappa_{l}$. 


\section{Numerical Method}

The perturbed equations and the boundary conditions comprise a 13th-order eigenvalue system. We use a $N$ order Chebyshev polynomial to expand each variable, the system of equations is required to solve for $8 N+1$ unknowns, including $8 N$ spectral coefficients and an interface deflexion $\eta$. A Chebyshev Tau method is used to solve the general eigenvalue problem. Details of the numerical procedure to solve the problem can be found in our previous work (Liu and Liu 2006) or Orzag's work (Orzag 1971). We have checked our calculation procedure and computational program by comparing our result with the analytical result reported by Pearson (1958) for the classical Marangoni problem. Our result is in good agreement with pearson's (Pearson 1958).

\section{Results and Discussion}

The goal of this paper is to investigate the influence of evaporation on the instability of the system. In order to do this, we will seek the Marginal curve of the Marangoni number versus the wave number. We chose the system consisting of a water layer in contact with its own vapor at $100^{\circ} \mathrm{C}$. The depth of the water layer is $1 \mathrm{~mm}$. The physical parameters are the same with Ozen's (Ozen and Narayanan 2004). The ratios of physical properties are $v^{*}=71.72, \rho^{*}=$ $6.25 \times 10^{-4}, \chi^{*}=3.68 \times 10^{-2}, \kappa^{*}=0.118$. The Prandtl number $\operatorname{Pr}=1.78$.

The physics of evaporation is complicated. The interfacial equilibrium degree, Rayleigh effect and Marangoni effect will play role in determining the instability of the system. When investigating the problem, we will look at different aspects of the problem separately whenever possible.

\section{Influence of Accommodation Coefficient $\beta$}

In order to focus on effect of the interfacial equilibrium degree on the Marangoni convection, we leave out the influence of gravitation. So, in the computations we set the acceleration of gravity $g=0$ and the evaporation velocity $w_{v 0}=0$.

We begin by presenting the physics of evaporation and the Marangoni convection. A fluctuation of temperature will result in a local surface tension gradient. Surface tension gradients at the interface act as tangential stress on adjacent fluids. The thermocapillarity provides a coupling of temperature and velocity at the interface. The interface relaxes at the point where a positive temperature disturbance created and the asso- ciated tangential stresses then induce radially divergent surface fluid motion. Continuity of the fluid requires a vertical ascending flow below the point. If the fluid is heated from below, the lower fluid is hotter than that near the interface. The uprising fluid driven by the surface tension will make the interfacial temperature increase. Consequently, the surface tension at that point keeps on decreasing, thus the convection is sustained. Considering a perturbed evaporation at the hot point, local latent heat consumption leads to intensive cooling of the liquid interface. Consequently, the positive temperature disturbance is reduced by local evaporation and the system gets more stable. For a larger $\beta$, this cooling effect is more intense and significantly stabilize the system.

Figure 2 shows the marginal curves of the Marangoni number versus the wave number for different accommodation coefficients. Non-volatile case $(\beta=0)$ is the non-equilibrium limit. In this case, the system reduces to the classical Marangoni case without evaporation. The Marginal curve of $\beta=0$ is similar to the classical Marangoni curve for a pure liquid. In the case of $\beta>0$, a new branch presents in the long wave region. Such a long wave branch corresponds the interfacial instability induced by evaporation effect. For the non-equilibrium limit $(\beta=0)$, the interfacial instability disappears. As show in Fig. 2 for $\beta>0$, the long wave instability is more unstable than the short wave instability corresponding to Marangoni convection. However, this long wave instability can be avoided in many physical

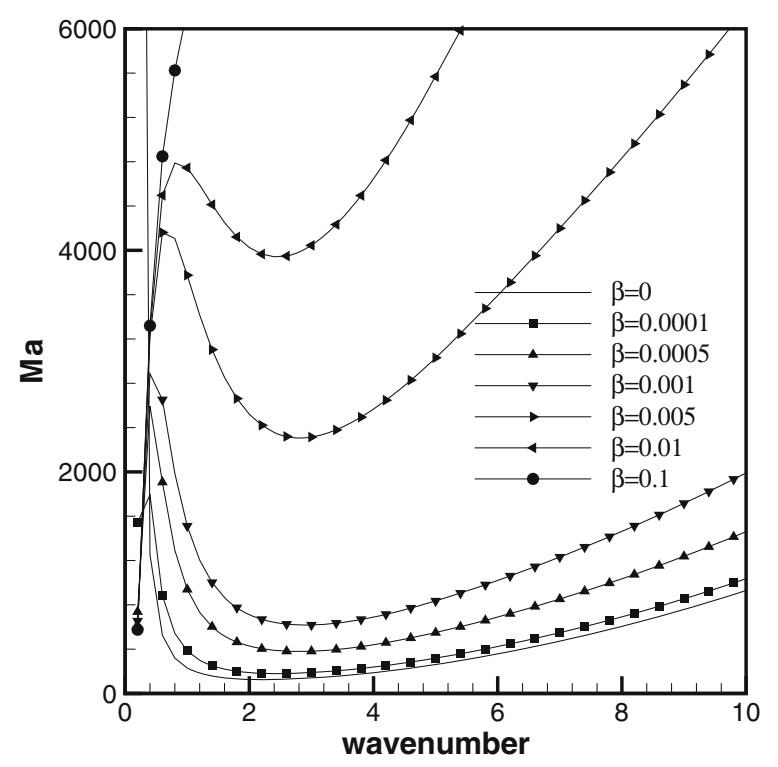

Fig. 2 The vapor liquid depth ratio is 1:10,the critical Marangoni number versus the wave number for various evaporation coefficients, $g=0$ 


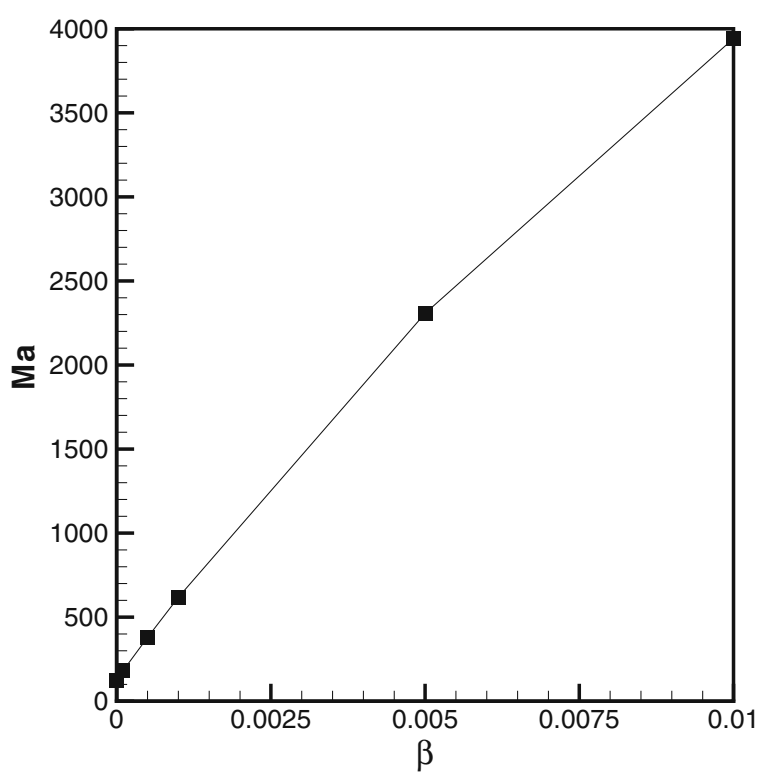

Fig. 3 The critical Marangoni number versus evaporation coefficients in the short wave range, the vapor depth ratio is $1: 10, g=0$

situations that involve a liquid layer with finite horizon dimensions. Since our interest focuses on the influence of evaporation on the Marangoni convection, the interfacial instability is not discussed in detail in this paper. For Marangoni convection, the system is most unstable for $\beta=0$. Physically speaking, the accommodation efficient $\beta$ presents the cooling ability of evaporation at the interface. Evaporation will lead to intensive cooling of the interface. This cooling mechanism reduces the temperature fluctuation at the interface and makes the system more stable. Figure 3 shows that the critical Marangoni number in the short wave branch and the accommodation coefficient have approximatively a linear relation. The greater is $\beta$, the more temperature difference across the liquid layer is need to sustain the Marangoni convection.

\section{Two Typical Coupling Modes}

For $\beta=0$, the system reduces to the non-volatile case. No evaporation occurs at the interface, so the liquid and the vapor are coupled only by heat conduction and surface tension. Figure 4a shows the convection pattern of Marangoni mode. The Marangoni convection initiates in the liquid layer. Near the interface, the vapor responds by being dragged, generating counter-rotating rolls in each layers.

For $\beta=0.01$, evaporation disturbance can occur at the interface. Figure 5a shows convection pattern of the evaporation mode. The liquid evaporates from the hot point and the vapor condenses at the cool point.
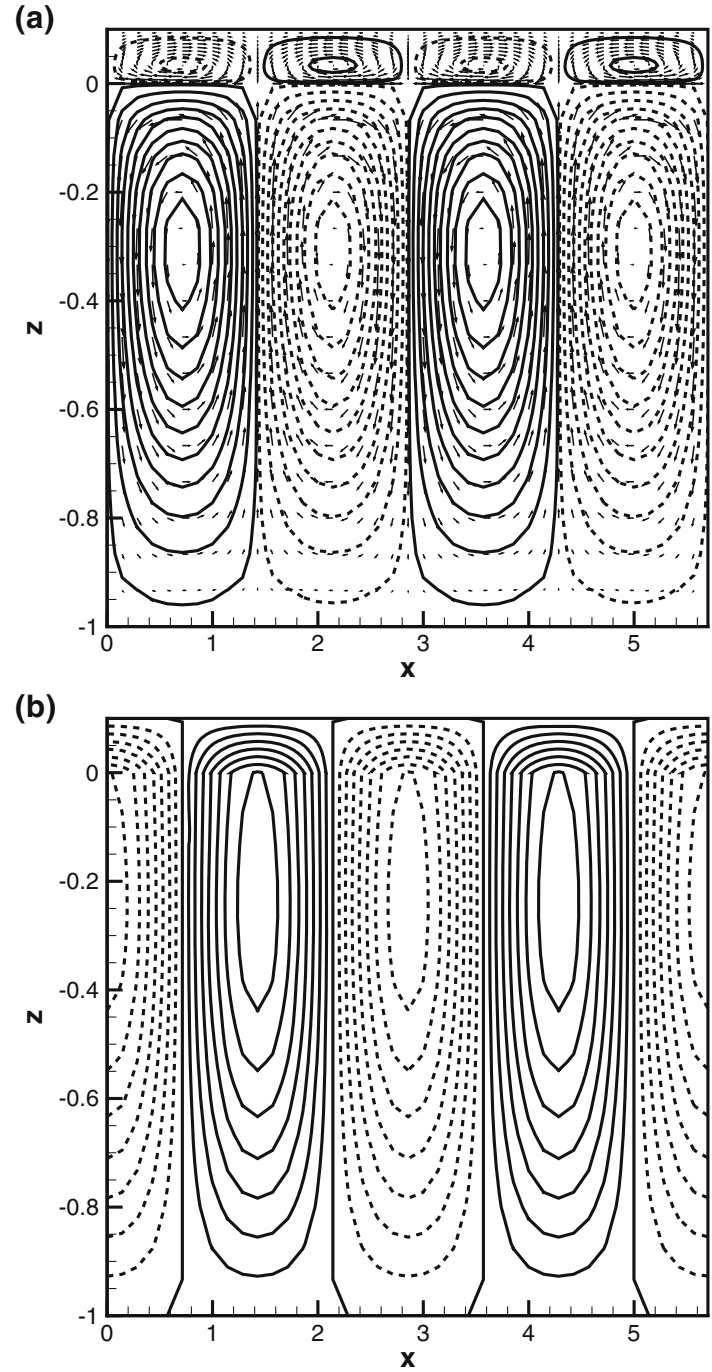

Fig. 4 Marangoni convective mode: Streamline pattern and velocity field (a) and temperature pattern (b) for $\beta=0, h=0.1$

Although the vapor may be dragged by liquid near the surface, evaporation and condensation are dominant in the vapor layer. Consequently, in the vapor layer the stream lines start from a hot point and end to a cool point. In this case, convective circulation does not occur in the vapor layer.

The temperature patterns of the Marangoni mode and the evaporation mode are shown in Fig. $4 \mathrm{~b}$ and Fig. 5b, respectively. In Fig. 4b, the isothermal lines penetrate the combined layers. The center of isothermal pattern locates near the interface. This result indicates that for non-evaporation case, the maximum temperature disturbance occurs near the interface when Marangoni convection occurs. Whereas, as shown in Fig. 5b, the temperature disturbance is near zero in the vapor layer and the center of temperature pattern locates beneath the interface. The temperature distur- 

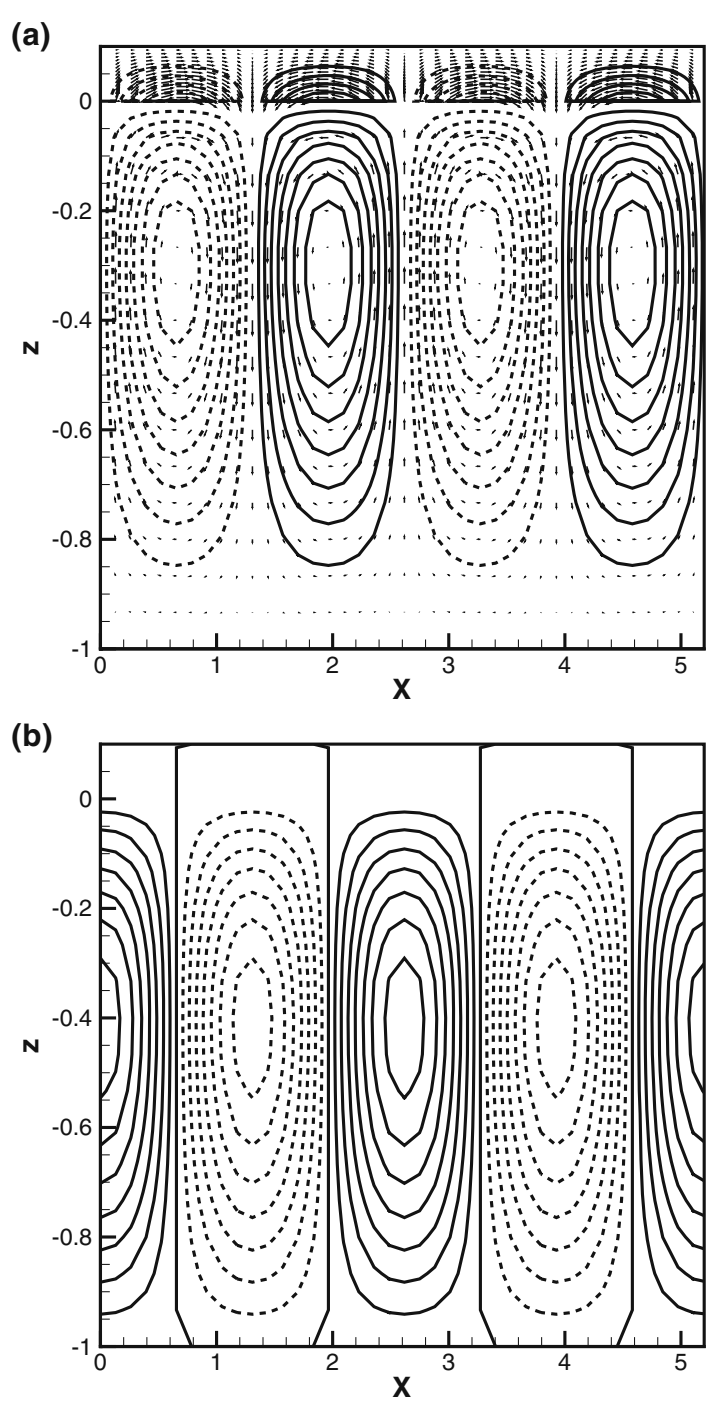

Fig. 5 Velocity field for $\beta=0.01, h=0.1(\mathbf{a}, \mathbf{b})$

bance at the interface is reduced by evaporation, so a greater temperature difference is needed to sustain the convection when evaporation occurs.

\section{The Influence of the Gravity}

In many laboratory situations, gravity plays an important role. Gravity has dual effects on the instability of the system, i.e. the Rayleigh effect (buoyancy-driven) and the interfacial effect. Buoyancy-driven convection occurs when a fluid is subjected to a temperature gradient perpendicular to the interface. The Rayleigh effect will destablilize the system. However, this effect can be avoid when the liquid layer is very thin. In this case, gravity simply pulls the perturbed interface back to its original position. In consequence, gravity reduces the interfacial instability and makes the system more stable.
In order to investigate the influence of gravity on the instability of a system under non-equilibrium evaporation, we consider three similar physical situations. In the first case, the gravity is left out and the Marangoni effect is retained. In this case, the convection can only be caused by the Marangoni effect and the evaporation. In the second case, the Rayleigh effect is taken into account and the interfacial effect of gravity is left out. In the third case, both the Rayleigh effect and the interfacial effect of gravity are taken into account. In order to compare the instability of these three systems, we define two parameters.

$R_{1}=M a_{R a} / M a, R_{2}=M a_{g} / M a$

$M a, M a_{R a}$ and $M a_{g}$ are the Marangoni numbers for the first, the second and the third cases, respectively.

In Fig. $6 R_{1}$ and $R_{2}$ merge in the long wave and small wave regions. In the long wave region, $R_{1}$ and $R_{2}$ are greater than 1 . This result indicates that the interfacial effect of gravity mainly plays role in the long wave region and makes the system more stable. In the range $2.0-8.0, R_{1} \simeq 1$ and $R_{2}$ is less than 1 . This means that the Rayleigh effect mainly operatives at medium wave region. In short wave region, $R_{1}$ and $R_{2}$ converge to 1. This result indicates that the interfacial effect has no influence on the instability of the system in short wave region.

Ozen and Narayanan (2004) investigated the influence of gravity on the instability for a quasi-equilibrium system. In their work, the minimum of $R_{2}$ is about 0.6. In our result, the minimum of $R_{2}$ is 0.9 . This difference

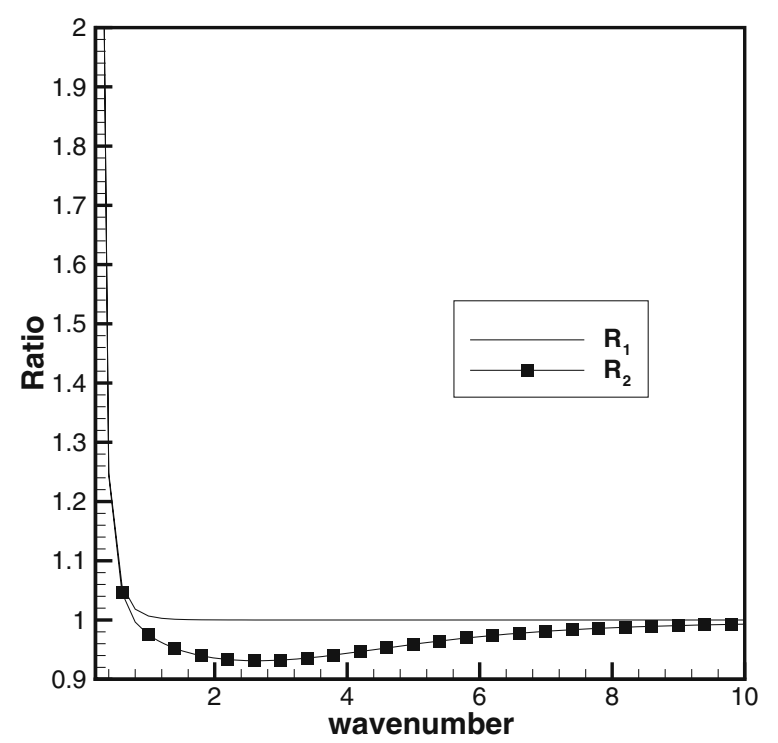

Fig. 6 The ratios $R_{1}$ and $R_{1}$ versus wavenumber, the depth ratio is $1: 10$, accommodation coefficient $\beta=0.01$ 
shows that the Rayleigh effect is more obvious for an equilibrium system.

\section{Conclusion}

The influence of evaporation on the RayleighMarangoni convection is investigated in the framework of linear instability analysis. We investigate the influence of the Marangoni effect, the Rayleigh effect, the dynamics of the vapor,and the degree of nonequilibrium at the interface on the instability of the system. It is shown that for a vapor-liquid system, long wave interfacial instability will occur when $\beta>0$. Only in the non-volatile limit $(\beta=0)$, the interfacial instability disappears. In the case of $\beta>0$, the minimum of the long wave branch is smaller than that of the short wave branch which corresponding to Marangoni convection. However, such a results does not mean the Marangoni convection will not occur in real situations. We should note that the horizontal domain is assumed to be infinite in this paper. In real conditions, finite horizontal geometry may prevent the long wave mode instability and the Marangoni mode may be preferred.

When $\beta>0$, the flow pattern is different to the nonvolatile case. The liquid evaporates at the hot point and condense at the cool point. Whereas, for non-volatile case, the vapor circulates in the upper domain.

Our results show that the gravity have dual effects on the system. The Rayleigh effect will destabilize the system and the interfacial effect of the gravity will stabilize the system.

Acknowledgements The authors thank the financial support for this research from National Natural Sciences Foundation of China (Grant Nos. 10772185 and 50890182) and the Knowledge Innovation Program of Chinese Academy of Sciences (KGCXSW-409).

\section{References}

Bénard, H.: Les tourbillons cellulaires dans une nappe liquide. Rev. Gen. Sci. Pures Appl. 11, 1261 (1900)

Block, M.J.: Surface tension as the cause of Bénard cells and surface tension deformation in a liquid film. Nature 178, 650 (1956)

Burelbach, J.P., Bankoff, S.G., Davis, S.H.: Nonlinear stability of evaporating/condensing liquid films. J. Fluid Mech. 195, 463 (1988)

Colinet, P., Legros, J.C., Velarde, M.G.: Nonlinear Dynamics of Surface-Tension-Driven Instabilities. Wiley-VCH, Berlin (2001)

Fang, G., Ward, C.A.: Temperature measured close to the interface of an evaporating liquid. Phys. Rev. E 59, 417 (1999)

Kennard, E.K.: Kinetic Theory of Gases. McGraw-Hill, New York (1938)

Liu, R., Liu, Q.S.: Linear stability analyses of convection in two-layer system with an evaporating vapor-liquid interface. Acta Mech. Sin. 22, 109 (2006)

Margerit, J., Colinet, P., Lebon, G., Iorio, C.S., Legros, J.C.: Interfacial nonequilibrium and Bénard-Marangoni instability of a liquid-vapor system. Phys. Rev. E 68, 041601 (2003)

Miller, C.A.: Stability of moving surface in fluid systems with heat and mass transport. Part II-Combined effects of transport and density difference between phases. AIChE 19, 909 (1973)

Nield, D.A.: Surface tension as the cause of Bénard cells and surface deformation in a liquid film. J. Fluid Mech. 19, 341 (1964)

Orzag, S.A.: Accurate solution of the Orr-Sommerfeld stabiltiy equation. J. Fluid Mech. 50, 689 (1971)

Ozen, O., Narayanan, R.: The physics of evaporative and convective instabilities in bilayer systems: linear theory. Phys. Fluids 16(12), 4644 (2004)

Palmer, H.J.: The hydrodynamic stability of rapidly evaporating liquids at reduced pressure. J. Fluid Mech. 75, 487 (1976)

Pearson, J.R.A.: On convection cells induced by surface tension. J. Fluid Mech. 4, 489 (1958)

Prosperetti, A., Plesset, M.S.: The stability of an evaporating liquid surface. Phys. Fluids 27(7), 1590 (1984)

Rayleigh, L.: On convection currents in a horizontal layer of fluid, when the higher temperature is on the under side. Phil. Mag. 32(6), 529 (1916) 